\title{
The lack of expression of the peripheral benzodiazepine receptor characterises microglial response in anaplastic astrocytomas
}

\author{
Shigetoshi Takaya ${ }^{1,2}$, Kazuo Hashikawa ${ }^{2}$, Federico E. Turkheimer ${ }^{3}$, Nicholas Mottram ${ }^{4}$, Manuel Deprez ${ }^{5}$, Koichi \\ Ishizu $^{6}$, Hidekazu Kawashima ${ }^{6}$, Haruhiko Akiyama ${ }^{7}$, Hidenao Fukuyama ${ }^{2}$, Richard B. Banati ${ }^{8}$, Federico \\ Roncaroli $^{4}$ \\ ${ }^{1}$ Radioisotope Research Centre, Kyoto University, Kyoto, Japan \\ ${ }^{2}$ Human Brain Research Centre, Kyoto University Graduate School of Medicine, Kyoto, Japan \\ ${ }^{3}$ Department of Clinical Neuroscience, Division of Neuroscience, Imperial College London, London, UK \\ ${ }^{4}$ University Department of Neuropathology, Division of Neuroscience and Mental Health, Imperial College London, Charing Cross Campus, \\ St Dunstans Road, London W6 8RP, UK \\ ${ }^{5}$ Department of Neuropathology, University of Liège, Liège, Belgium \\ ${ }^{6}$ Department of Nuclear Medicine and Diagnostic Imaging, Kyoto University Graduate School of Medicine, Kyoto, Japan \\ ${ }^{7}$ Tokyo Institute of Psychiatry, Tokyo, Japan \\ ${ }^{8}$ School of Medical Radiation Science, Ramaciotti Centre for Brain Imaging at the Brain Mind Research Institute [BMRI], University of \\ Sydney, Sydney, NSW, Australia
}

\begin{abstract}
The peripheral benzodiazepine receptor $(\mathrm{PBR})$ is a $18 \mathrm{kDa}$ molecule mainly involved in cholesterol transport through the mitochondrial membrane. In microglia, PBR is expressed from the earliest stages of activation and appears to exert a pro-inflammatory function. This molecule is commonly up-regulated in inflammatory, degenerative, infective and ischaemic lesions of the central nervous system but it has never been reported in glioma-infiltrating microglia. We examined two anaplastic astrocytomas showing minimal contrast-enhancement and therefore little damage of the blood brain barrier to minimise the presence of blood borne macrophages within tumour tissue. The two lesions were studied in vivo using positron emission tomography (PET) with the specific PBR ligand $\left[{ }^{11} \mathrm{C}\right](\mathrm{R})-\mathrm{PK} 11195$ and the corresponding tumour tissue was investigated with an anti-PBR antibody. Glioma-infiltrating microglia were characterised for molecules involved in antigen presentation and cytotoxic activity. As comparison, PBR was investigated in three brains with multiple sclerosis (MS) and three with Parkinson's disease (PD). The expression profile of four anaplastic astrocytomas was also exploited and results were compared to the profile of eleven samples of normal temporal lobe and nine cases of PD. PET studies showed that $\left[{ }^{11} \mathrm{C}\right](\mathrm{R})-\mathrm{PK} 11195$ binding was markedly lower in tumours than in the contralateral grey matter. Pathological investigation revealed that glioma-infiltrating microglia failed to express PBR and cytotoxic molecules although some cells still expressed antigen presenting molecules. PBR and cytotoxic molecules were highly represented in MS and PD. Evaluation of microarray datasets confirmed these differences. Our results demonstrated PBR suppression in glioma-infiltrating microglia and suggested that PBR may have a relevant role in modulating the anti-tumour inflammatory response in astrocytic tumours.
\end{abstract}

Keywords : Astrocytoma ; Microglia ; Peripheral benzodiazepine receptor ; PK11195 ; Positron emission tomography

\section{Introduction}

Microglia are the resident immune cells of the central nervous system (CNS) [1]. They are ubiquitously distributed across brain regions in a "resting" state and constitute a network of surveillance and defence cells, with their cytoplasmic processes continuously scanning the surrounding microenvironment [2]. Upon detection of any insult to brain tissue, microglial cells undergo a process of activation that entails changes in their shape and function. Cytoplasmic processes shorten and thicken until cells appear to be indistinguishable from bloodborne macrophages, begin producing a myriad of cell-signalling molecules and receptors, and acquire the capacity of antigen presentation, phagocytosis, migration and proliferation [3,4]. Activation can be triggered by 
a broad variety of stimuli including hypoxia, accumulation of insoluble molecules, infective agents or the contact with neoplastic cells [4]. In anaplastic astrocytomas and glioblastomas, microglia contribute significantly to the total tumour mass but the fact that neoplastic cells manage to survive and grow in their presence indicate the failure of normal defence mechanisms [5,6]. The causes of poor microglial response to tumour cells are still unclear although recent studies have demonstrated glioma-infiltrating microglial cells show an impairment of antigen presenting functions [6-10].

The peripheral benzodiazepine receptor (PBR) is one of the earliest and most commonly expressed molecules during microglial activation in inflammatory, degenerative, vascular and infective CNS diseases [3, 5, 11]. For this reason, high levels of PBR are generally considered a marker of microglial activation [5], and the PBRspecific ligand $\left[{ }^{11} \mathrm{C}\right](\mathrm{R})-\mathrm{PK} 11195$ is widely used in positron emission tomography (PET) studies to trace microglial infiltrate as an in vivo indicator of tissue damage [11]. Also known as PK-11195 binding protein or translocator protein, the PBR is an $18 \mathrm{kDa}$ molecule consisting of a five helices spanning the membrane lipid bilayer of the outer mitochondrial membrane and forming a heterotrimer together with a voltage dependent anion channel and an adenine nucleotide transporter $[12,13]$. The PBR is expressed in several normal tissues with the highest levels in gonads and cortical cells of adrenal gland $[14,15]$ and its major function is to actively transport cholesterol and proteins through the mitochondrial membrane [13]. The actual role of PBR in microglial cells is still to be elucidated although recent studies in vitro [16] and animal model [17] suggested that it may exert a pro-inflammatory function. Interestingly, several studies have found high-PBR levels in tumour cells of glioblastomas and to a lesser extent of anaplastic astrocytomas [18] but none of them have mentioned gliomainfiltrating microglial cells as possible source of PBR.

Here, we present the results obtained during a feasibility study designed to explore the expression of PBR in human astrocytic tumours and the diagnostic use of $\left[{ }^{11} \mathrm{C}\right](\mathrm{R})$-PK11195 PET-scan in neuro-oncology. We investigated PBR in vivo using $\left[{ }^{11} \mathrm{C}\right](\mathrm{R})-\mathrm{PK} 11195$ PET imaging and with immunohistochemistry in tumour tissue. Tumour-infiltrating microglial cells were also characterised for the expression of molecules involved in antigen presentation and cytotoxic activity and results obtained in astrocytomas were compared with cases of multiple sclerosis (MS) and Parkinson's disease (PD), which are known to contain a substantial amount of activated microglia.

\section{Clinical findings}

\section{Case 1}

This 27-year-old male presented with a 2-month history of seizures. MR-scan (1.5 Tesla Sonata scanner, Siemens, Erlangen, Germany) demonstrated a lesion of the right temporal lobe, which appeared inhomogeneously hypointense on T1-weighted sequences and hyperintense in the T2-weigh ted sequences. Only minimal enhancement was observed after administration of gadolinium (Fig. la). No therapy has been given prior to surgery but $800 \mathrm{mg} /$ day of valproate.

\section{Case 2}

This 53-year-old female presented with a 4-year history of progressive dysesthesia in right upper and lower extremities. MRI-scan revealed a right fronto-insular intra-axial lesion that was inhomogeneously hypointense in T1-weighted images and hyperintense in the T2-weighted sequences (Fig. 1c). Only mild diffuse enhancement was seen. No radiotherapy, chemotherapy or corticosteroid therapy were administered prior to the PET scanning and surgical resection.

Both patients underwent surgical debulking and samples were submitted for pathological examination. This study was approved by the Ethics Committee of the Kyoto University Graduate School of Medicine (No. 420) and by the Central Ethics Committee, London UK (No 05/Q0401/3), and informed consent was obtained from both patients. 
Fig. 1 Gadolinium-enhanced MRI $(a, c)$ and $\left[{ }^{11} C\right](R)$-PK11195 PET superimposed on 3D MRI $(b, d)$. The intensity of the lesions is low in the T1-weighted images with minimal contrast enhancement (a, patient 1, left frontal region; c, patient 2, left frontotemporal region; arrowheads). Both lesions show decreased $\left[{ }^{11} C\right](R)-$ PK11195 binding than the normal grey matter ( $b, d$; BP binding potential)

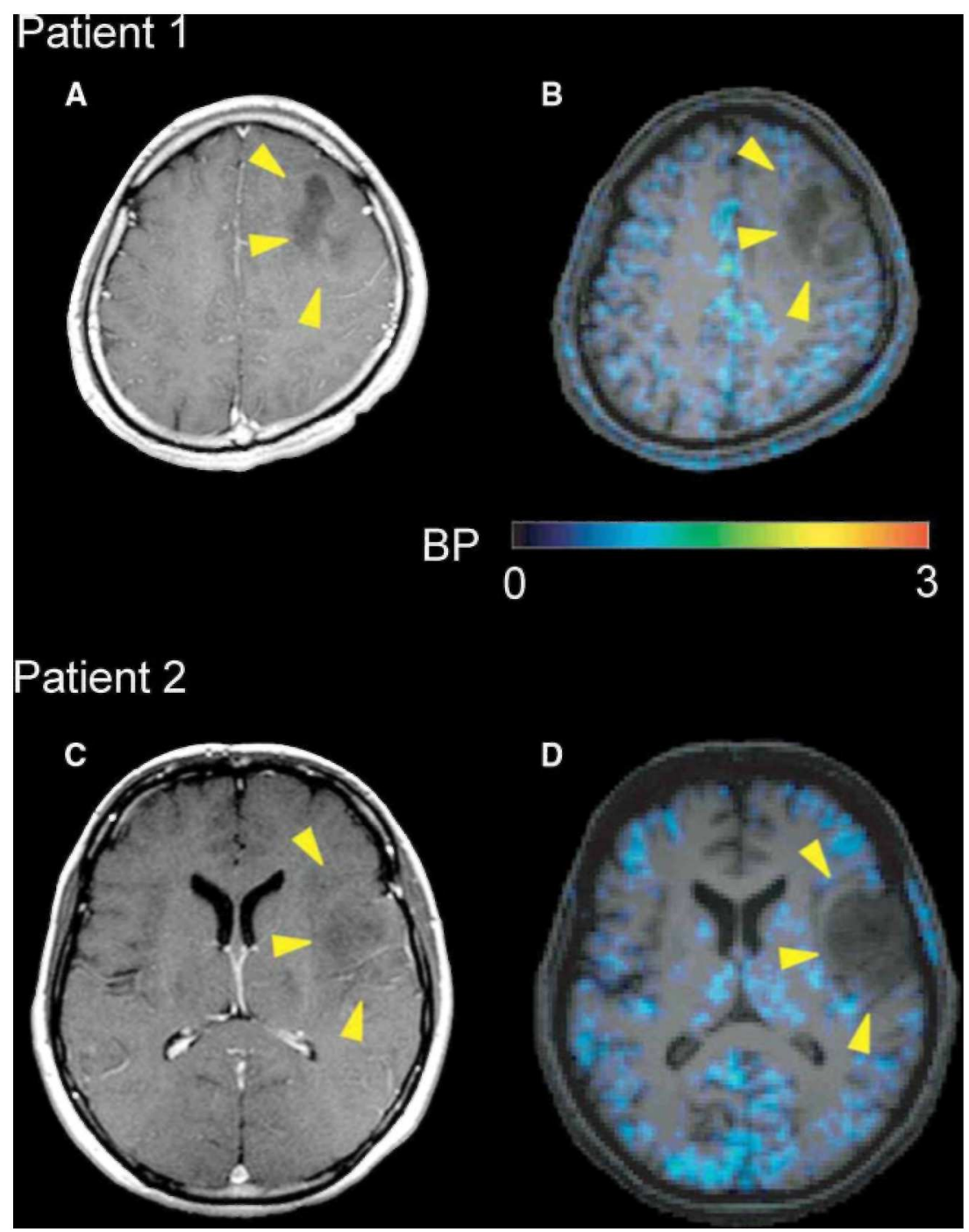

\section{Materials and methods}

\section{PET imaging acquisition and analysis}

Both patients underwent $\left[{ }^{11} \mathrm{C}\right](\mathrm{R})-\mathrm{PK} 11195$ PET scan (Advance, General Electric, Milwaukee, WI, USA) and a three-dimensional T1-weighted MRI (magnetisation prepared rapid acquisition gradient-echo sequence; TR/TE, 2000/4.38; 3 Tesla Trio scanner, Siemens) prior to surgery. PET study was performed in three-dimensional acquisition mode as previously reported [19]. A $6 \mathrm{mCi}$ bolus of $\left[{ }^{11} \mathrm{C}\right](\mathrm{R})-\mathrm{PK} 11195$ was injected intravenously into the patients $30 \mathrm{sec}$ after the acquisition scan started. Dynamic PET data were acquired over $60 \mathrm{~min}$ as 18 temporal frames. Attenuation correction factors were determined using a 15-min eight-emission scan. Data were reconstructed with a ramp filter. Kinetic modelling was performed by Logan graphical analysis without blood sampling [20]. We used the time-activity course of the radioactivity in a reference tissue (normal grey matter contralateral to the tumour) of each patient as the input function. The binding potential (BP) was calculated as $\mathrm{BP}=\mathrm{DVR}-1$, where DVR is the ratio of the volume of distribution (DV) in the region of interest to that in the reference region. The DVR was calculated as the slope of the Logan plot that was obtained by linear regression analysis. Finally, PET images were coregistered onto 3D MRI images by using the mutual information algorithm implemented in SPM2 (Wellcome Department of Imaging Neuroscience, UCL, London, UK). 


\section{Pathological investigation}

Surgical samples were fixed in $10 \%$ buffered formalin and paraffin embedded. Five-micron sections were cut and stained with haematoxylin-eosin (HE). Immunohistochemistry was performed using the avidin-biotin complex/ peroxidase (ABC) method with antibodies directed against PBR (monoclonal clone 8D7; dilution 1:200; kindly supplied by Dr. Pierre Casellas, Department of Immunology-Oncology, Sanofi Synthelabo, Montpellier, France), MHC class II antigen (HLA-DR) (MHCII) (Dako Cytomaton, Glostrup, Denmark; clone CR3/43; dilution 1:200), CD80 (B7.1) (Abeam, Cambridge, UK, clone MAB104 dilution 1:100), CD86 (B7.2) (Sigma, Deisenhofen, Germany, clone B72-H2, dilution 1:100), Tumour Necrosis Factor alpha (TNF-alpha) (Chemicon, Termecula, CA, USA, clone 195, dilution 1:200), inducible nitric oxide synthase (iNOS) (R\&D, Wiesbaden, Germany, clone 2B2-D2, dilution 1:50) and cyclooxigenase 2 (COX-2) (BD Transduction Lab, Palo Alto, CA, USA, clone 33, dilution 1:250). For all antibodies, the sections were pretreated in $1 \mathrm{mM}$ EDTA (pH 8) for $20 \mathrm{~min}$ in microwave oven at $500 \mathrm{~W}$.

The epitope recognised by the anti-PBR antibody corresponding to the cholesterol binding domain (amino acids 158-169) [12, 21] was compared with all the known human proteins (Blast/Fasta; www.pir.georgetown.edu) and no match was found other than with the three PBR isoforms. The protocol for PBR was assessed using normal adrenal gland that constitutively contains high level of the protein [15]. We also tested the anti-PBR antibody with Western-blot analysis with extracts from adrenal gland and normal frontal cortex. A standard protocol was followed [22].

The extent of expression of microglial PBR in non-neoplastic CNS diseases was examined in active demyelination from three post-mortem brains with MS, and in the substantia nigra of three brains with PD. Brains with MS were donated to the UK MS Tissue Bank and PD cases were donated to the UK PD Society Tissue Bank, both at Imperial College, London. These samples were previously immunostained for MHCII antigen and they were known to contain activated microglial cells. Lymphocytes, neutrophils, endothelial cells and smooth muscle cells present within tumour tissues were considered internal positive controls, as they are known to constitutively express PBR [13, 23].

\section{Evaluation of microarray datasets}

The differences between microglial in astrocytomas and non-neoplastic CNS diseases were also explored by examining the expression profile of four anaplastic astrocytomas, which are part of a group of gliomas we have studied with expression microarrays (Affymetrix, Santa Clara, CA, USA, U-133 Plus 2; available online GEO database, GEO submission GSE2817) [for details see reference 24]. Tumours were compared with eleven normal temporal lobes removed in course of surgery for intractable epilepsy. Anaplastic astrocytomas and normal temporal lobes were characterised for the extent of microglial infiltrate using MHCII antigen and for the expression of PBR and COX-2 (immunohistochemical stains are described above). As example of a nonneoplastic CNS condition, we explored a dataset obtained from the substantia nigra of nine PD cases (Affymetrix, U-133 A) [25]. Cases of PD were compared with the expression profile of the substantia nigra of seven age-matched normal brains. The three PD cases used for immunohistochemistry were part of the group used for expression microarrays. We investigated the expression of molecules involved in microglial response including MHC class II antigen (HLA-DR), CD40, CD80 (B7.1), CD86 (B7.2), CD40, Toll-like receptors (TLR) 1-8 and 10, and TNF-alpha receptors 1 . Astrocytomas and PD cases were compared to the respective control tissue. Details on microarray data processing and normalisation are reported in the respective references [24, 25]. Statistical analysis was performed using a Mann-Whitney test. Analysis of expression profiles did not include other molecules contributing to microglial immune response such as TNF-alpha, iNOS and COX-2 because probes for these molecules are not contained in the Affymetrix arrays.

\section{Results}

\section{PET findings}

$\left[{ }^{11} \mathrm{C}\right](\mathrm{R})-\mathrm{PK} 1195$ PET studies revealed that the binding of the ligand was markedly lower in both tumours than in the contralateral grey matter [Figs, 1b, 1d] [Fig. 2]. The tumour/normal grey matter ratios of the integrated radioactivity and $\mathrm{BP}$ in each patient were 0.67 (patient 1 ) and 0.51 (patient 2), and 0.68 (patient 1) and 0.51 (patient 2), respectively. 
Fig. 2 Regional time activity curves (TAC) extracted from the dynamic $\left[{ }^{11} C\right](R)-P K 11195$ PET data of both patients (no decay correction). In both cases, the TAC is lower in astrocytomas than that in the reference cortical region

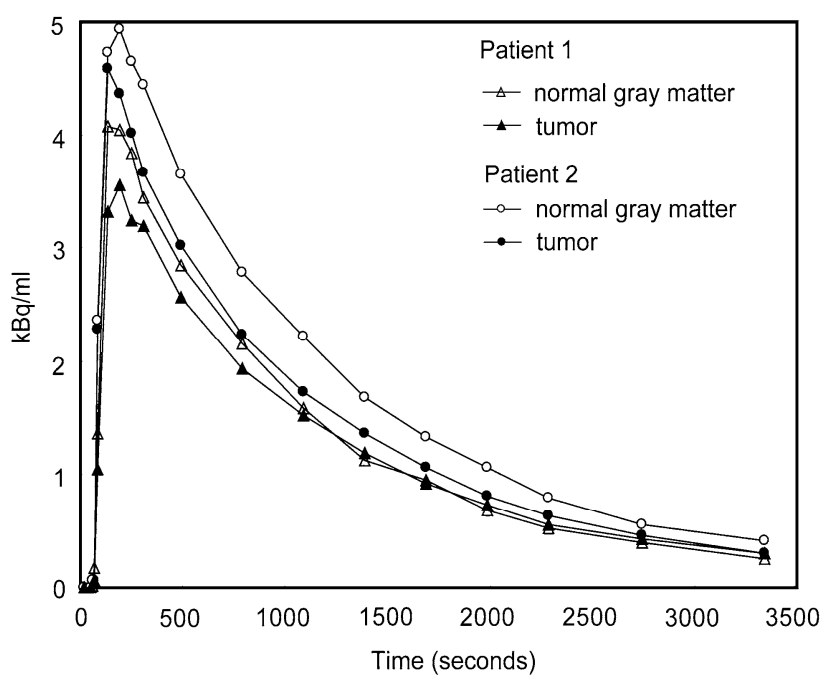

Validation of the anti-PBR monoclonal antibody

Normal adrenal gland demonstrated expression of PBR in the cells of the zona glomerulosa, reticularis and fasiculata and the sections of normal brain showed expression in a few perivascular macrophages. Western-blot analysis revealed a band of $18 \mathrm{kDa}$ in both extracts corresponding to the known molecular weight of the PBR. Signal seen in brain tissue was much weaker than signal in adrenal gland.

\section{Pathological findings}

Both tumours were diagnosed as anaplastic astrocytoma (grade III, WHO classification) [26] by two independent neuropathologists. They were composed of astrocytic cells with fibrillary cytoplasm and moderate to severe nuclear pleomorphism. Mitotic activity was brisk in both cases. No necrosis and, vascular and endothelial proliferation was seen (Fig. 3a). As seen with the immunoreaction for MHCII, both tumours contained a substantial number of activated microglial cells and macrophages that accounted for $-40 \%$ of the total number of cells (Fig. 3b). The anti-PBR antibody demonstrated expression in a few tumour cells and no expression in microglia (Fig. 3c) PBR-positive lymphocytes were observed within the vessels and leptomeninges (Fig. 3d). Leptomeningeal arteries showed PBR expression in endothelium and smooth muscle cells of the tunica media whereas normal vessels entrapped with tumours vessels showed weak expression. In both cases, some microglial cells expressed CD80 and CD86. TNF-alpha was present in a few microglial cells mainly located in the subpial tissue whereas iNOS and COX-2 were only observed in a few neoplastic cells.

The three cases of MS showed intense PBR expression in activated microglial cells surrounding active plaques as well as in microglia and macrophages within areas of demyelination (Fig. 4a). Strong expression of PBR was also seen in activated microglia in the substantia nigra of the three PD cases (Fig. 4b). In all the samples examined we observed PBR expression in the wall of several medium sized and small arteries. Both MS and PD cases featured expression of CD86, TNF-alpha, iNOS and COX-2 in microglial cells.

\section{Evaluation of microarray datasets}

Immunohistochemical stains for MHCII revealed a diffuse microglial infiltrate in the four anaplastic astrocytomas used for microarrays analysis compared to normal control tissue. The PBR was expressed in tumour cells and in a few microglial cells. Notably, all the tumours showed a considerably high number of MHCII-positive microglial cells than PBR-positive and COX-2 positive microglia. Expression analysis of anaplastic astrocytomas showed levels of PBR as low as control brains. Expression of MHCII, CD86 and TLRs 4 and 5 in anaplastic astroctyomas were significantly higher than normal brains $(P<0.1)$ whereas CD80 and CD40 were high but expression values did not reach significance. TNF-alpha receptor 1 was only mildly 
elevated and TLRs 1-3, 6-8 and 10 were as low as normal brain. In the substantia nigra of PD cases, the expression of PBR, TLRs 4 and 5 and TNF-alpha 1 receptor were increased compared to normal nigra $(P<0.1)$; MHCII, CD40, CD80 and CD86 showed borderline levels compared to controls. All values of expressions were normalised to the respective control tissue (Fig. 5).

Fig. 3 The tumour in patient 1 shows the features of an anaplastic fibrillary astrocytoma $(a ;$ HE staining; $\times 10)$. The immunoreaction for the MHC class II antigen demonstrates a considerable number of activated microglia $(b, \times 10)$. PBR is expressed in a few tumour cells but it is undetectable in microglia (c; patient $1 \times 25)$. PBR expression is observed in the leptomeningeal lymphocytes and leptomeningeal vessels $(d$, patient $1, \times 4)$
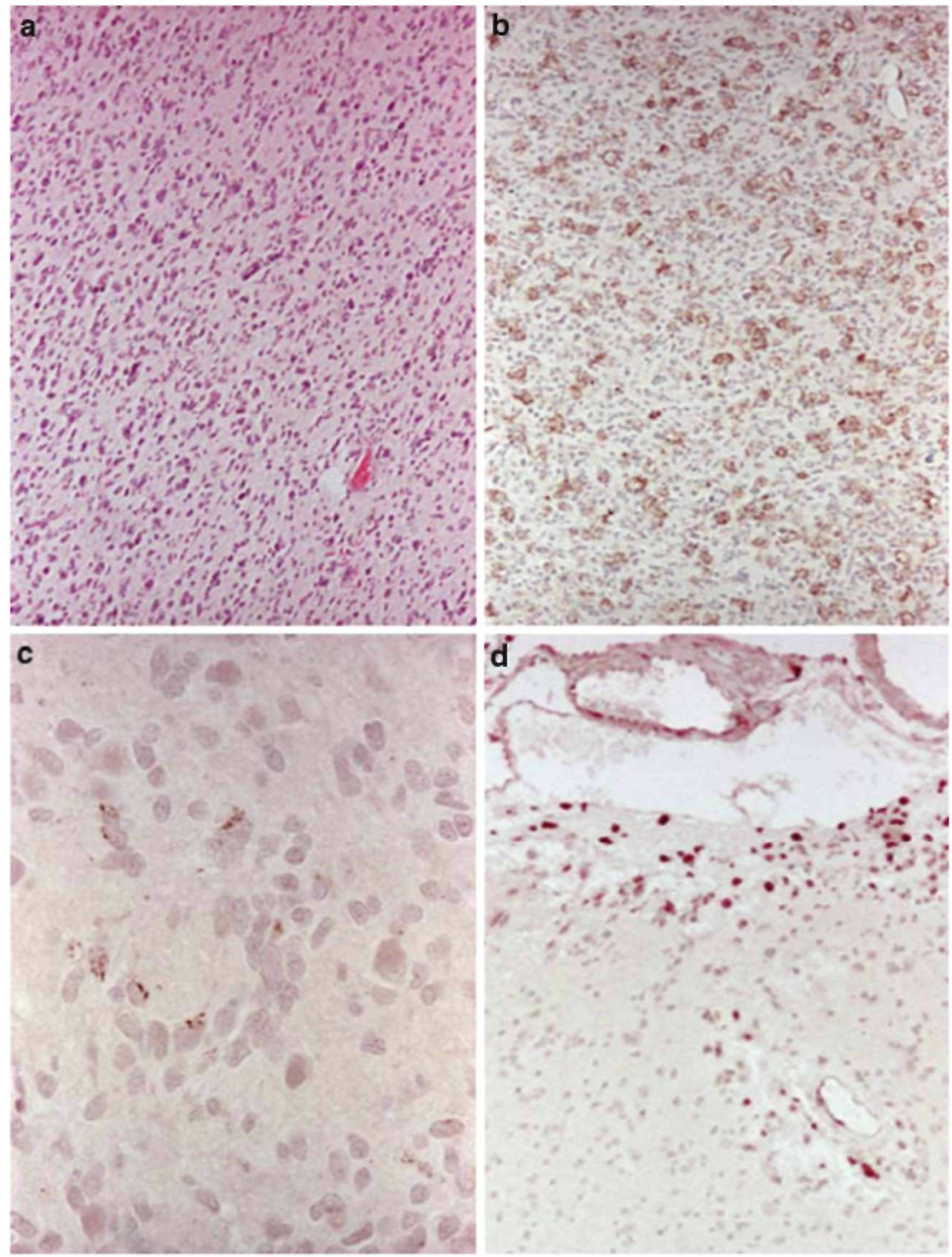
Fig. 4 Intense expression of PBR is seen in activated microglia in an active MS plaque (a) and Parkinson's disease ( $b$, nigra) (ABC/ peroxidase)

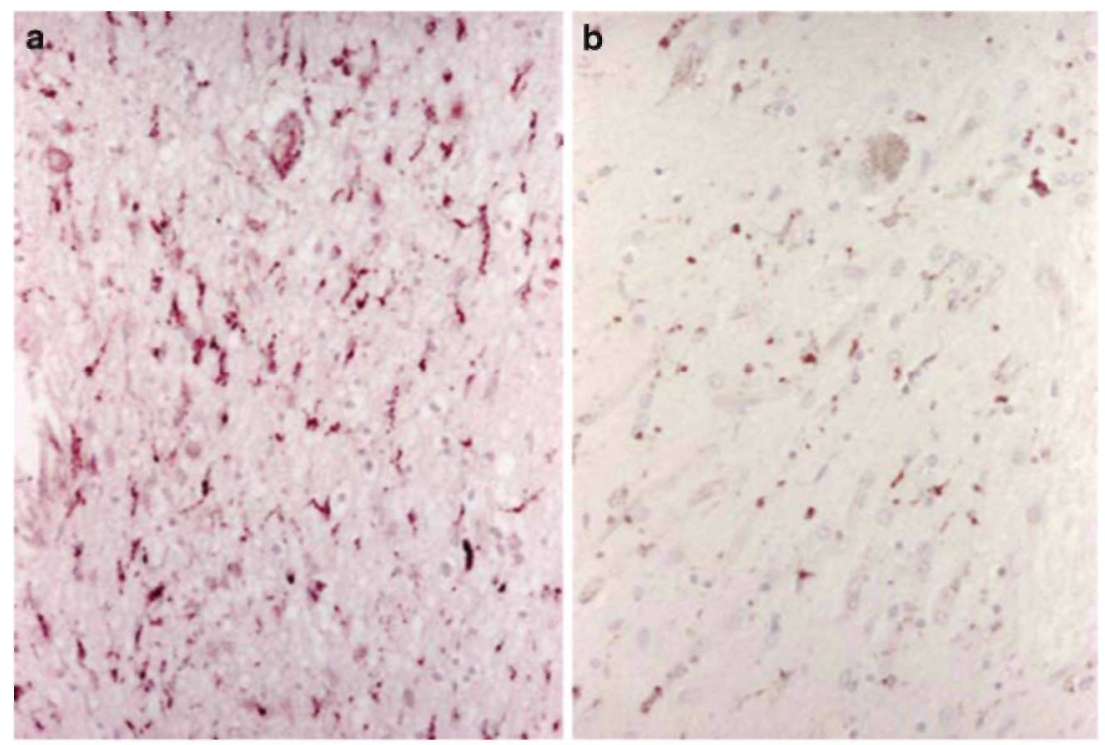

Fig. 5 This diagram demonstrates the expression of mRNA of PBR, MHCII, CD86, TLR4 and 5 and TNF alpha receptor 1 in anaplastic astrocytomas (blue) and PD (green). Expression is normalised to the control tissue

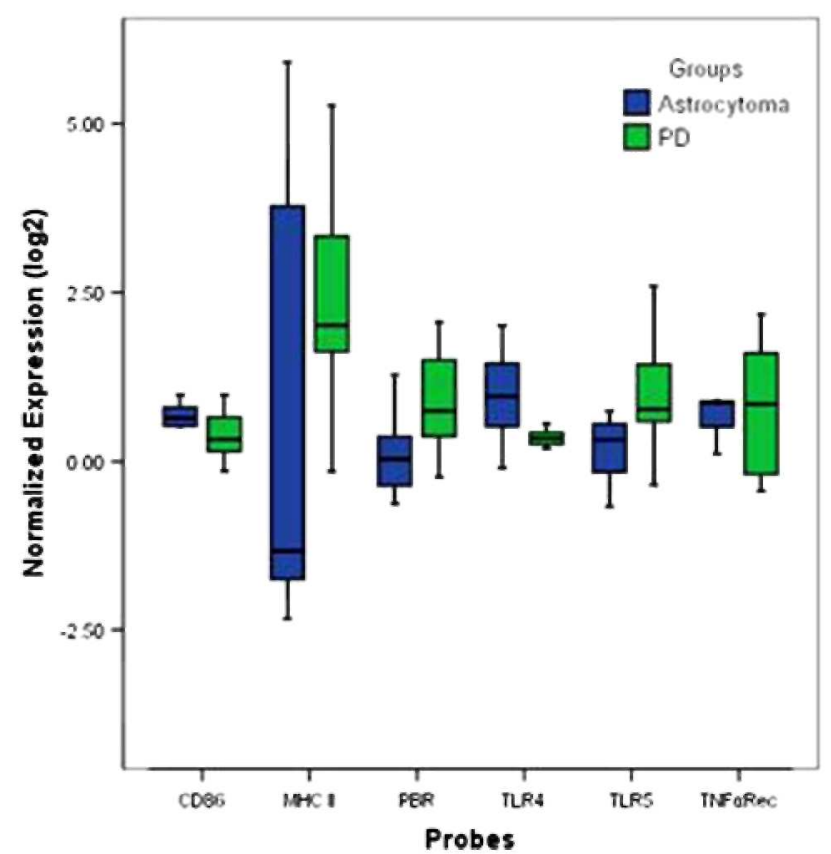

\section{Discussion}

We observed that activated microglial cells infiltrating two cases of anaplastic astrocytoma do not express PBR. This finding is interesting because it differs from all imaging studies with [ $\left.{ }^{11} \mathrm{C}\right](\mathrm{R})-\mathrm{PK} 11195[11]$, microarray studies [5], studies in vitro [27, 28], animal model [3, 29, 30] and post-mortem brains [22] that showed high expression of PBR in activated microglia in a variety of inflammatory, degenerative, infective and vascular CNS disorders. Our approach of combining the evaluation of PBR using $\left[{ }^{11} \mathrm{C}\right](\mathrm{R})$-PK11195 PET-scan and immunohistochemistry offered the advantage of studying the binding capacity of this molecule in vivo and, on 
tissues its distribution in the cell types constituting the two astrocytomas without the changes that may occur examining microglia in vitro, outside the complexity of interactions with other cell types [5]. In addition, these two cases represented a rare opportunity and an excellent model to investigate microglial PBR because they showed low-PBR expression in neoplastic cells and minimal contrast enhancement indicating minimal disruption of the blood brain barrier and therefore little presence of blood-borne macrophages within tumour tissue.

We first excluded that the lack of PBR in glioma-infiltrating microglia depended on the techniques used. Time activity curves showed good perfusion of the radioligand indicating that low BP seen in PET studies did not depend on reduced blood flow within tumours. The dose of unlabelled PK11195 injected for PET imaging along with the labelled ligand was too low to saturate binding sites. Positive immunohistochemical reactions obtained with adrenal gland, MS and PD confirmed that the anti-PBR antibody 8D7 is suitable for routinely processed tissue and the search in protein Blast/Fasta, and Western-blot analysis confirmed its specificity. Finally, PBR immunolabelling in tumour cells and intravascular and leptomeningeal mononuclear inflammatory cells that normally produce PBR $[13,23]$ indicated good preservation of the antigen in tumour samples. Taken altogether, these findings confirmed that the very low-PET signal and the lack of immunolabelling in tumour tissue were consistent with a very low expression of PBR in tumour tissue.

In the present two cases only a minority of neoplastic cells expressed PBR. The presence of a limited number of PBR-positive tumour cells was essential to our study because it allowed us for a better evaluation of its expression in microglial cells. Previous studies that investigated PBR in human astrocytomas using autoradiography with [ $\left.{ }^{3} \mathrm{H}\right]$ PK11195 [31-38] or immunohistochemistry [18, 39-41] observed that the great majority of glioblastomas have high-PBR expression whereas anaplastic astrocytomas show variable expression with some lesions showing PBR levels as high as low-grade examples and normal brain [39]. The function of PBR in tumour cells is still unclear but some authors suggested that this molecule may have a role in inducing proliferation and regulating apoptosis [41]. Interestingly, none of these studies reported intra-tumoural microglial cells and macrophages to be a source of PBR. Using autoradiography, Benavides et al. [32] examined PBR expression in glioblastomas, MS, brain infarct and normal brains. They observed a considerable increase in PBR expression in all lesions and a spatial correlation between increased expression of PBR and accumulation of macrophages in MS plaques and infarcts but not in glioblastomas where PBR expression was reportedly limited to tumour cells.

Our results obtained with the two cases studied with PET-scan combined with the results of expression microarrays showed that glioma-infiltrating microglia in anaplastic astrocytomas express very low levels or no PBR and that glioma-infiltrating microglia have impaired cytotoxic activity (lack of TNF-alpha, COX-2 and iNOS) although they retain antigen presenting functions, at least in some cells (expression of CD86 and TLRs 4 and 5). In addition, we demonstrated that microglial response in anaplastic astrocytomas substantially differs from the response in non-neoplastic conditions such as MS and PD. These findings elicit an intriguing hypothesis on the role of PBR in microglial immune functions. The PBR is one of the most common molecules expressed by microglial cells during activation but its role in these cells is unknown and the molecular pathway linking the functions of PBR to microglial response has not been elucidated [5]. In a mouse model, Banati et al. [42] showed that PBR is up-regulated at the early stages of activation when microglial cells still show a ramified morphology and suggested that PBR may be a key molecule in microglial immune response. Recent in vitro studies demonstrated that binding of PBR with the antagonist PK-11195 can suppress lipopolysaccaride-induced expression of TNF-alpha and COX-2 [16] and that PK-11195 may block store-operated channels mediated $\mathrm{Ca}^{2+}$ influx and the production of COX-2 induced by platelet-activating factor [43]. These results indicate that PBR may exert a pro-inflammatory activity and suggest a correlation between modulation of mitochondrial PBR and cellular inflammatory response with store-operated channels acting as the molecule that links the two pathways. In human glioblastomas, Hussain et al. [6] observed that glioma-infiltrating microglia and macrophages separated from tumour cells up-regulate MHCII and TLRs 1, 2, 3 and 4, show very low levels of CD86 and lack of CD80 and TNF-alpha, even after stimulation with lipopolysaccaride. Finally, previous studies in glial tumours did not document expression of COX-2 and iNOS in glioma-infiltrating microglia although they showed expression in tumour cells and their increase with tumour grade [44, 45]. If these and our results are considered altogether, we may speculate that down-regulation of PBR in anaplastic astrocytomas can interfere with the immune response of glioma-infiltrating microglia by suppressing cytotoxic activity. Interestingly, He et al. [46] documented very low expression of TNF-alpha and iNOS in activated microglia surrounding brain metastatic non-small cell lung carcinomas suggesting that mechanisms that lead to impairment of microglial anti-tumour activity may be common in primary and secondary CNS tumours.

Positron emission tomography studies performed here showed that $\left[{ }^{11} \mathrm{C}\right](\mathrm{R}) \mathrm{PK} 11195$ binding in both astrocytomas was markedly lower than in the contralateral grey matter. Turkheimer et al. [47] recently reported 
that normal brains show a baseline up-take of $\left[{ }^{11} \mathrm{C}\right](\mathrm{R}) \mathrm{PK} 11195$ due to the presence of PBR in the tunica media and endothelium of intra-parenchymal arteries. In the current study, immunohistochemical stains demonstrated lack of microglial PBR in normal vessels entrapped within neoplastic tissue but still preserved expression in leptomeningeal vessels. Such reduction of the baseline expression of PBR in vessels justifies a binding of $\left[{ }^{11} \mathrm{C}\right]$ (R) PK11195 in astrocytomas lower than normal grey matter. In addition, absent expression of PBR in microglia, low expression in neoplastic cells and intra-tumoural vessels might suggest that molecules present in tumour microenvironment can down-regulate PBR.

Two previous studies have investigated PBR expression in human gliomas by using $\left[{ }^{11} \mathrm{C}\right](R)$-PK11195 PET [48, 49]. Junck et al. [48] examined three low-grade and three anaplastic astrocytomas, three glioblastomas and one case defined as indeterminate grade. They observed detectable signal in eight lesions but only 60 and $80 \mathrm{~min}$ after injection and not in the early images. In addition, tumour/grey matter ratio, tumour/white matter ratio and tumour radioactivity did not correlate with tumour grade. Their findings are not comparable to ours because these authors did not use the R-enantiomer of PK11195, which exhibits maximum contrast at $\sim 10$ min after injection approaching the equilibrium thereafter. Moreover, many lesions investigated in their study showed contrast enhancement and therefore damage of the blood brain barrier. Disruption of the blood brain barrier allows blood-borne mononuclear cells to infiltrate tumour tissue and such a recruitment of haematogenous cells that highly express PBR may give confounding results. The results obtained by Pappata et al. [49] were in keeping with the known kinetic properties of $\left[{ }^{11} \mathrm{C}\right](\mathrm{R}) \mathrm{PK}-11195$ but a parallel with our findings is also difficult because they studied a case of glioblastoma, hence a lesion with significant damage of the blood brain barrier.

In conclusion, we have shown that activated microglial cells infiltrating anaplastic astrocytomas may not express PBR as they do in neuroinflammatory, neurodegenerative, vascular and infective CNS diseases. Our results suggest that PBR has an important in modulating the inflammatory microglial response against tumour cells and highlight the complexity of the in vivo response of microglia in astrocytic tumours. Absence of PBR in microglial cells and in the wall of intra-tumoural vessels suggests that tumour microenvironment may contain molecules capable of inducing down-regulation of PBR. Further studies are required to elucidate the role of PBR in microglial immune response and the causes leading to its suppression in high-grade astrocytomas.

\section{Acknowledgements}

This study was supported by a Grant-in-Aid for Scientific Research on Priority Areas (17022023) from the Ministry of Education, Culture, Sports, Science and Technology, Japan. Dr. Roncaroli's research is partly supported by the Charity BRTC. We thank Dr. Nobuhiro Mikuni from the Department of Neurosurgery at the Kyoto University Graduate School of Medicine for recruiting the patients, Ms. Lynne Christian from the Department of Neuropathology at the Imperial College in London for critically reviewing the manuscript, Professor Graeber and Dr. Moran, Department of Neuropathology, Imperial College for kindly making available their dataset generated with cases of Parkinson's Disease.

\section{References}

1. Kreutzberg GW (1996) Microglia: a sensor for pathological events in the CNS. Trends Neurosci 19:312-318

2. Nimmerjahn A, Kirchhoff F, Helmchen F (2005) Resting microglial cells are highly dynamic surveillants of brain parenchyma in vivo. Science 308:1314-1318

3. Schwartz M, Butovsky O, Brack W et al (2006) Microglial phenotype: is the commitment reversible? Trends Neurosci 29:68-74

4. Gebicke-Haerter PJ (2005) Microarrays and expression profiling in microglia research and in inflammatory brain disorders. J Neurosci Res 81:327-341

5. Hussain SF, Yang D, Suki D et al (2006) The role of human glioma-infiltrating microglia/macrophages in mediating antitumour immune responses. Neurooncology 8:261-279

6. Roggendorf W, Strupp S, Paulus W (1996) Distribution and characterization of microglia/macrophages in human brain tumours. Acta Neuropathol (Berl) 92:288-293

7. Tran CT, Wolz P, Egensperger R et al (1998) Differential expression of MHC class II molecules by microglia and neoplastic astroglia: relevance for the escape of astrocytoma cells from immune surveillance. Neuropathol Appl Neurobiol 24:293-301

8. Schartner JM, Hagar AR, Van HM et al (2005) Impaired capacity for upregulation of MHC class II in tumour-associated microglia. Glia $51: 279-285$ 
Published in : Journal of neuro-oncology (2007), vol. 85, pp. 95-103

Status : Postprint (Author's version)

9. Town T, Nikolic V, Tan J (2005) The microglial "activation" continuum: from innate to adaptive responses. J Neuroinflammation 2:24

10. Watters JJ, Schartner JM, Badie B (2005) Microglia function in brain tumours. J Neurosci Res 81:447-455

11. Banati RB (2002) Visualising microglial activation in vivo. Glia 40:206-217

12. Papadopoulos V, Baraldi M, Guilarte TR et al (2006) Translocated protein (18 kDa): new nomenclature for the peripheral-type benzodiazepine receptor based on its structure and molecular function. Trends Pharmacol Sci 27:402-409

13. Veenman L, Gavish M (2006) The peripheral benzodiazepine receptor and the cardiovascular system. Implications for drug development. Pharmacol Ther 110:502-524

14. Gavish M, Bachman I, Shoukrun R et al (1999) Enigma of the peripheral benzodiazepine receptor. Pharmacol Rev 51:629-650

15. Bribes E, Carriere D, Goubet C et al (2004) Immunohistochem-ical assessment of the peripheral benzodiazepine receptor in human tissues. J Histochem Cytochem 52:19-28

16. Choi HB, Khoo C, Ryu JK et al (2002) Inhibition of lipopolysaccharide-induced cyclooxygenase-2, tumour necrosis factor-alpha and $[\mathrm{Ca} 2+] \mathrm{i}$ responses in human microglia by the peripheral benzodiazepine receptor ligand PK11195. J Neurochem 83:546-555

17. Farges RC, Torres SR, Ferrara P et al (2004) Involvement of steroids in anti-inflammatory effects of peripheral benzodiazepine receptor ligands. Life Sci 74(11):1387-1395

18. Han Z, Slack RS, Li W et al (2003) Expression of peripheral benzodiazepine receptor (PBR) in human tumours: relationship to breast, colorectal, and prostate tumour progression. J Recept Signal Transduct Res 23:225-238

19. Turkheimer HE, Aston JA, Banati RB et al (2003) A linear wavelet filter for parametric imaging with dynamic PET. IEEE Trans Med Imaging 22(3):289-301

20. Logan J, Fowler JS, Volkow ND et al (1996) Distribution volume ratios without blood sampling from graphical analysis of PET data. J Cereb Blood Flow Metab 16:834-840

21. Dussossoy D, Carayon P, Feraut D et al (1996) Development of a monoclonal antibody to immuno-cytochemical analysis of the cellular localization of the peripheral benzodiazepine receptor. Cytometry 24:39-48

22. Banati RB, Newcombe J, Gunn RN et al (2000) The peripheral benzodiazepine binding site in the brain in multiple sclerosis: quantitative in vivo imaging of microglia as a measure of disease activity. Brain 123:2321-2337

23. Cahard D, Canat X, Carayon P et al (1994) Subcellular localization of peripheral benzodiazepine receptors on human leukocytes. Lab Invest 70:23-28

24. Turkheimer FE, Roncaroli F, Hennuy B et al (2006) Chromosomal patterns of gene expression from microarray data: methodology, validation and clinical relevance in gliomas. BMC Bioinformatics 7:526-544

25. Duke DC, Moran LB, Turkheimer FE et al (2004) Microglia in culture: what genes do they express? Dev Neurosci 26:30-37

26. Kleihues P, Louis DN, Scheithauer BW et al (2002) The WHO classification of tumours of the nervous system. J Neuropathol Exp Neurol 61:215-225

27. Wilms H, Claasen J, Rohl C et al (2003) Involvement of benzodiazepine receptors in neuroinflammatory and neurodegenerative diseases: evidence from activated microglial cells in vitro. Neurobiol Dis 14:417-424

28. Moran LB, Duke DC, Turkheimer FE et al (2004) Towards a transcriptome definition of microglial cells. Neurogenetics 5:95-108

29. Kuhlmann AC, Guilarte TR (2000) Cellular and subcellular localization of peripheral benzodiazepine receptors after trimethyltin neurotoxicity. J Neurochem 74:1694-1704

30. Chen MK, Baidoo K, Verina T et al (2004) Peripheral benzodiazepine receptor imaging in CNS demyelination: functional implications of anatomical and cellular localization. Brain 127:1379-1392

31. Olson JM, Junck L, Young AB et al (1988) Isoquinoline and peripheral-type benzodiazepine binding in gliomas: implications for diagnostic imaging. Cancer Res 48:5837-5841

32. Benavides J, Cornu P, Dennis T et al (1988) Imaging of human brain lesions with an omega 3 site radioligand. Ann Neurol 24:708-712

33. Ferrarese C, Appollonio I, Frigo M et al (1989) Benzodiazepine receptors and diazepam-binding inhibitor in human cerebral tumours. Ann Neurol 26:564-568 
Published in : Journal of neuro-oncology (2007), vol. 85, pp. 95-103

Status : Postprint (Author's version)

34. Black KL, Ikezaki K, Santori E et al (1990) Specific high-affinity binding of peripheral benzodiazepine receptor ligands to brain tumours in rat and man. Cancer 65:93-97

35. Broaddus WC, Bennett JP Jr (1990) Peripheral-type benzodiazepine receptors in human glioblastomas: pharmacologic characterization and photoaffinity labeling of ligand recognition site. Brain Res 518:199-208

36. Cornu P, Benavides J, Scatton B et al (1992) Increase in omega 3 (peripheral-type benzodiazepine) binding site densities in different types of human brain tumours. A quantitative autoradiography study. Acta Neurochir (Wien) 119:146-152

37. Takada A, Mitsuka S, Diksic M et al (1992) Autoradiographic study of peripheral benzodiazepine receptors in animal brain tumour models and human gliomas. Eur J Pharmacol 228:131-139

38. Miyazawa N, Hamel E, Diksic M (1998) Assessment of the peripheral benzodiazepine receptors in human gliomas by two methods. J Neurooncol 38:19-26

39. Miettinen H, Kononen J, Haapasalo H et al (1995) Expression of peripheral-type benzodiazepine receptor and diazepam binding inhibitor in human astrocytomas: relationship to cell proliferation. Cancer Res 55:2691-2695

40. Brown RC, Degenhardt B, Kotoula M et al (2000) Location-dependent role of the human glioma cell peripheral-type benzodiazepine receptor in proliferation and steroid biosynthesis. Cancer Lett 156:125-132

41. Vlodavsky E, Soustiel JF (2007) Immunohistochemical expression of peripheral benzodiazepine receptors in human astrocytomas and its correlation with grade of malignancy, proliferation, apoptosis and survival. J Neurooncol 81(1):1-7

42. Banati RB, Myers R, Kreutzberg GW (1997) PK ('peripheral benzodiazepine')-binding sites in the CNS indicate early and discrete brain lesions: microautoradiographic detection of [3H]PK11195 binding to activated microglia. J Neurocytol 26:77-82

43. Hong SH, Choi HB et al (2006) Mitochondrial ligand inhibits store-operated calcium influx and COX-2 production in human microglia. J Neurosci Res 83:1293-1298

44. Shono T, Tofilon PJ, Bruner JM et al (2001) Cyclooxygenase-2 expression in human gliomas: Prognostic significance and molecular correlations. Cancer Res 61:4375-4381

45. Hara A, Okayasu I (2004) Cyclooxigenase 2 and inducible nitric oxide synthase in human astrocytic gliomas: correlation with angiogenesis and prognostic significance. Acta Neuropathol 108:43-48

46. He BP, Wang JJ et al (2006) Differential reactions of microglia to brain metastases of lung cancer. Mol Med 12:161-170

47. Turkheimer FE, Edison $\mathrm{P}$ et al (2007) Reference and target region modelling of $\left[{ }^{11} \mathrm{C}\right]-(R)-\mathrm{PK} 11195$ brain studies. J Nucl Med 48:158167

48. Junck L, Olson JM et al (1989) PET imaging of human gliomas with ligands for the peripheral benzodiazepine binding site. Ann Neurol 26:752-758

49. Pappata S, Cornu P et al (1991) PET study of carbon-11-PK 11195 binding to peripheral type benzodiazepine sites in glioblastoma: a case report. J Nucl Med 32:1608-1610 